\title{
Bone metastases: an overview
}

\author{
Filipa Macedo, ${ }^{1}$ Katia Ladeira, ${ }^{2}$ Filipa Pinho, ${ }^{2}$ Nadine Saraiva, ${ }^{1}$ Nuno Bonito, ${ }^{1}$ Luísa Pinto, ${ }^{2}$ \\ Francisco Gonçalves ${ }^{2}$
}

${ }^{1}$ Medical Oncology Department, Portuguese Oncology Institute, Coimbra; ${ }^{2}$ Internal Medicine Department, Braga Hospital, Braga, Portugal

\begin{abstract}
Bone is a frequent site of metastases and typically indicates a short-term prognosis in cancer patients. Once cancer has spread to the bones it can rarely be cured, but often it can still be treated to slow its growth. The majority of skeletal metastases are due to breast and prostate cancer. Bone metastasis is actually much more common than primary bone cancers, especially in adults. The diagnosis is based on signs, symptoms and imaging. New classes of drugs and new interventions are given a better quality of life to these patients and improved the expectancy of life. It is necessary a multidisciplinary approach to treat patients with bone metastasis. In this paper we review the types, clinical approach and treatment of bone metastases.
\end{abstract}

\section{Introduction}

Metastasize is a process that involves loss of intercellular cohesion, cell migration, angiogenesis, access to systemic circulation, survival in circulation, evasion of local immune responses, and growth at distant organs..$^{1,2}$

Bone is the third most frequent site of metastasis, behind lung and liver. ${ }^{3}$ Prostate and breast cancer (BC) are responsible for the majority of the skeletal metastases (up to $70 \%$ ). ${ }^{4}$ This reflects both the high incidence and relatively long clinical course of these

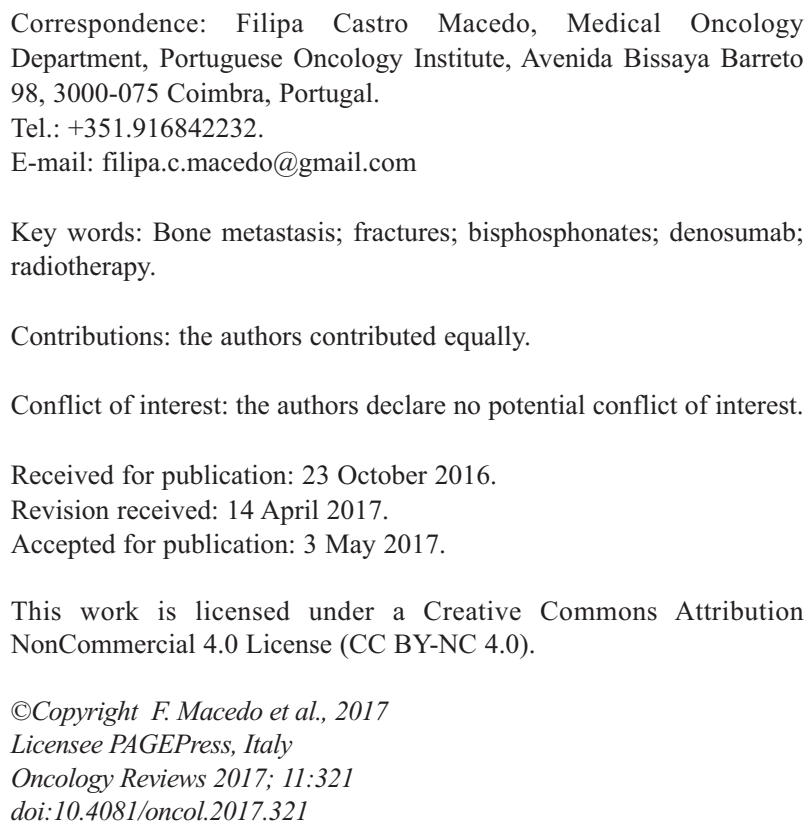

tumors. The overall incidence of bone metastasis is not known. ${ }^{3}$ The relative incidence of bone metastasis by type of tumor, in patients with advanced metastatic disease, is: $65-75 \%$ in $\mathrm{BC}$; $65-$ $75 \%$ in prostate; $60 \%$ in thyroid; $30-40 \%$ in lung; $40 \%$ in bladder; $20-25 \%$ in renal cell carcinoma and $14-45 \%$ in melanoma. The median-survival from diagnosis of bone metastasis is: 6months in melanoma; 6-7 months in lung; 6-9 months in bladder; 12 months in renal cells carcinoma; $12-53$ months in prostate; $19-25$ months in $\mathrm{BC}$ and 48 months in thyroid. ${ }^{5}$

Bone metastases are a major cause for morbidity, characterized by severe pain, impaired mobility, pathologic fractures, spinal cord compression, bone marrow aplasia and hypercalcemia. ${ }^{4}$

\section{Types of bone metastasis}

Bone metastasis are classified as osteolytic, osteoblastic or mixed, according to the primary mechanism of interference with normal bone remodeling:

- Osteolytic, characterized by destruction of normal bone, present in multiple myeloma (MM), renal cell carcinoma, melanoma, non-small cell lung cancer, non-hodgkin lymphoma, thyroid cancer or langerhans-cell histiocytosis. The great majority of $\mathrm{BC}$ produces osteolytic metastases. This bone destruction is primarily mediated by osteoclasts and not a direct effect of tumor cells. ${ }^{5,6}$ Other way, with lesser importance, is the compression of vasculature and consequent ischaemia in the late stages of cancer. ${ }^{3}$ Parathyroid hormonerelated peptide (PTHrP) has a major role in the development of osteolytic lesions. ${ }^{7}$ It is unclear if bone microenvironment induces cancer cells to express PTHrP or if cells that metastasize to bone have an intrinsic higher PTHrP expression. ${ }^{8}$ The receptor activator of NF-kappaB ligand (RANKL) play a critical role in the formation of osteoclasts by stimulating precursor cells when binds to receptor activator of NF-kappaB (RANK) on the cell membrane of osteoclast precursors. ${ }^{9}$

- Osteoblastic (or sclerotic), characterized by deposition of new bone, present in prostate cancer, carcinoid, small cell lung cancer, Hodgkin lymphoma or medulloblastoma. The mechanisms of osteoblastic metastases are still poorly understood. In some instances the new bone formation is not necessarily preceded by bone resorption. ${ }^{3}$ Transforming growth factor, bone morphogenic proteins (BMP) and endothelin-1 are associated with osteoblast generation. ${ }^{10}$ Prostate-specific antigen (PSA), can cleave PTHrP, allowing the osteoblastic reaction predominate by decreasing bone reabsorption. ${ }^{11}$ Core binding factor alpha1 (Cbfa1), also known as Runx-2, is linked to osteoblast differentiation. ${ }^{12}$

- Mixed, if a patient has both osteolytic and osteoblastic lesions, or if an individual metastasis has both osteolytic and osteoblastic components, present in $\mathrm{BC}$, gastrointestinal can- 
cers and squamous cancers. Although BC gives origin predominantly to osteolytic lesions, $15-20 \%$ of women have osteoblastic lesions, or both type of lesions. ${ }^{13}$

\section{Mechanisms of metastases to bone}

Bone metastasis are almost always multiple and involve axial skeleton. ${ }^{4}$ It has been suggested that this distribution might be in relation to the hematopoietically active red bone marrow. ${ }^{13}$ There exists a paravertebral network that may play a role in the development of bone metastasis. ${ }^{14}$ This theory is supported by the high incidence of bone metastases without corresponding lesions in the lung (suggest an alternative pathway of spread). In addiction, the microenvironment must be favorable for tumor cell survival. ${ }^{4}$

Once the tumor cell is in circulation, it needs:

Vascular adhesion and extravasation: the cell interacts with endothelium in order to extravasate and stay in a specific tissue. ${ }^{15}$ Chemoattractive and adhesion molecules play a fundamental role in this selective retention of cancer cells in bone marrow vasculature. Cancer cells use equivalent molecules to vascular cell adhesion molecules (VCAM) and E-selectin to adhere to endothelium. ${ }^{16,17} \mathrm{We}$ also know that chemokines, integrins, osteopontin, bone sialoprotein and type I collagen are critical for organ colonization by cancer cells. ${ }^{18,19}$ Examples of such interactions are: expression of CXCR4 by neuroblastoma tumors that mediates the attachment to stromalcell derived factor 1 in bone (SDF-1 or CXCL12); ${ }^{20}$ expression of RANK by BC that mediates the attachment to RANKL in bone; ${ }^{21}$ expression of sialoprotein by non-small cell lung cancer that facilitate binding to collagen type I in bone. ${ }^{22}$

- Micro-environmental support: The seed-and-soil hypothesis tells us that the microenvironment provides a fertile ground (the soil), for the survival and growth of metastatic cancer cells (the seed). ${ }^{23}$ The bone formation and reabsorption release and activate survival and growth promoting factors that may contribute to bone metastases development. ${ }^{24}$

Epithelial - Mesenchymal transition: Normal cell can lose their epithelial features and acquire mesenchymal characteristics. This process is called Epithelial-Mesenchymal Transition, and enables epithelial cells to migrate to a new environment. This occurs mainly during embryogenesis, but in cancer cells this process confers the invasive phenotype. ${ }^{4}$

\section{Clinical aspects}

The median survival of patients after first bone metastasis by $\mathrm{BC}$ is 20 months. This is in marked contrast to those with metastases of $\mathrm{BC}$ in the liver, 3 months, or with bone metastases from non-small cell lung cancer, 6 months. ${ }^{25,26}$

In prostate cancer, men with a good performance status and bone only disease, affecting predominantly the axial skeleton, have a median duration of disease control after androgen blockade of 4years and a median survival of 53 months. This is in marked contrast to those with visceral disease with a median survival of 30 months and 12 months with visceral disease and poor performance status. $^{27}$

Bone metastases are a major cause for morbidity, characterized by severe pain, impaired mobility, pathologic fractures, spinal cord compression, bone marrow aplasia and hypercalcemia. ${ }^{4}$

Hypercalcaemia is the most common metabolic complication of malignant disease. ${ }^{28}$ It is frequent in squamous-cell carcinomas of the lung, $\mathrm{BC}$ and kidney, MM and lymphoma. The four main contributes are: focal osteolysis by tumor cells, generalized osteolysis by humoral factors secreted by the tumor, increased renal tubular reabsorption of calcium and impaired renal glomerular function. BC secretes PTHrP; MM leads to impaired renal function due to deposition of Bence-Jones proteins; some lymphomas produce active metabolites of vitamin-D, which increases both bone resorption and intestinal absorption of calcium. ${ }^{28}$

Moderate to severe hypercalcaemia, if left untreated, causes a number of unpleasant symptoms related to dysfunction of the gastrointestinal tract, kidneys and central nervous systems such as constipation, polyuria, polydipsia and fatigue. In final stages, hypercalcemia can leads to cardiac arrhythmias and acute renal failure. ${ }^{3}$ With hypercalcaemia, parathyroid hormone levels are suppressed, and PTHrP may be elevated. This leads to increased osteoclastic bone resorption. Hypercalcaemia carries a poor prognosis with a median survival of $10-12$ weeks. ${ }^{5}$

Pathologic fractures occur in $10-30 \%$ of all cancer patients, with proximal parts of the long bones being the most frequent fracture site, and the femur accounting for over half of all cases. ${ }^{5}$ Rib fractures and vertebral collapses are also very common, which can lead to kyphoscoliosis and a degree of restrictive lung disease. ${ }^{29}$ $\mathrm{BC}$ accounts for $60 \%$ of the pathologic fractures and lung cancer for only $10 \%$ of cases. ${ }^{30,31}$

The most disability is caused by a long bone fracture or epidural extension of tumor into the spine. ${ }^{3}$ The probability of developing a pathological fracture increases with the duration of metastatic involvement. Although the intensity of bone pain is not directly associated with fracture risk, pain that is exacerbated by movement does appear to be an important factor in predicting impending fracture. Primary internal stabilization followed by radiotherapy is usually the treatment of choice. ${ }^{3}$

Bone pain is the most common type of pain from cancer, is poorly localized, worse at night, not necessarily relieve with sleep or lying down. ${ }^{32}$ In cancer patients, development of bone pain usually is considered to be highly suggestive of bone metastases. ${ }^{5}$ The pain associated with bone metastasis could be either of inflammatory or mechanical origin. Inflammatory pain is related to the local release of cytokines and chemical mediators by the tumor cells, periosteal irritation, stimulation of intraosseous nerves. Mechanical pain is related to the pressure or mass effect of the tumor tissue within the bone, with loss of bone strength thus turning into activity-related pain. ${ }^{5}$ The inhibition of osteoclastic bone reabsorption reduces bone pain. ${ }^{32}$ The use of osteoclast inhibitors, such bisphosphonates and denosumab, reduce bone pain.

The development of back pain in a patient with cancer, associated with an abnormal spinal radiograph should warn the physician for possible spinal cord compression. It is more commonly seen in BC (20-30\%) and lung cancer (15\%). ${ }^{5}$ For a successful rehabilitation the diagnosis must be fast, high-dose corticosteroids treatment, rapid assessment and urgent referral for both decompression and spinal stabilization. If compression is not relieved within 24$48 \mathrm{~h}$, neurologic recovery is unlikely. 3,28

A basic screening must be performed when one of the signs and symptoms described above are present: a complete blood-cell count to evaluate for anemia and myelosuppression; serum calcium, phosphorus, 25-hydroxyvitamin D, alkaline phosphatase, creatinine, thyroid-stimulating hormone, protein electrophoresis and parathyroid hormone level to identify bone turnover and evaluate hypercalcemia. ${ }^{5,33}$ This study must be complemented with imaging data. $^{5}$

Bone scintigraphy is highly sensitive but usually has a low specificity. Sensitivity of 99Tc scintigraphy has been reported to range from 62 to $89 \%$, with a false-positive rate as high as $40 \%$. It 
is more sensitive and more specific than plain films and computed tomography (CT) scans, while magnetic resonance imaging (MRI) is superior in evaluating vertebral metastases. ${ }^{34}$ It provides information on osteoblastic activity and skeletal vascularity, with preferential uptake of tracer at sites of active bone formation that reflects the metabolic reaction of bone to the disease process, whether neoplastic, traumatic or inflammatory. ${ }^{33}$

Radiographs are a fast, cheap, and readily available technique for evaluating bone metastases. Plain radiography should be the first test in the evaluation of bone pain. A plain radiography is very specific but sensitivity is low (44-50\%) because metastatic lesions may not appear on X-ray at initial stages. Lesions up to $1 \mathrm{~cm}$ might go undetected, while more than $50 \%$ of trabecular bone must be destroyed before it will be evident on film. Medullary lesions are more difficult to detect than lesions in cortical bone because of the limited contrast in trabecular bone. ${ }^{35}$ Osteolytic lesions appear as a darker hole in the gray-white bone image; osteoblastic lesions appear as spots that are whiter than the bone around them.

The sensitivity of $C T$ for the diagnosis of bone metastases ranges from $71-100 \% .{ }^{36} \mathrm{CT}$ produces images with excellent soft tissue and contrast resolution. Bone destruction and sclerotic deposits are usually clearly shown and any soft tissue extension of bone metastases is easily visualized. CT is particularly useful to localize lesions for biopsy. ${ }^{33}$

$M R I$ is required to diagnose spinal cord compression and is useful in imaging bone marrow to assess involvement by the tumor. The sensitivity ranges from $82-100 \%$ and its specificity ranges from $73-100 \% .{ }^{37}$

Positron emission tomography (PET) detects the presence of tumor directly by quantifying metabolic activity. It is superior to bone scintigraphy in the detection of bone metastases from lung cancer (sensitivity 92\%, specificity 99\%), ${ }^{38}$ and from BC (sensitivity $95 \%$, specificity 94\%). ${ }^{39}$ It has lower accuracy in renal and prostate cancer bone metastasis because they are slow growing (so, the uptake of 18 -fluorodeoxyglucose is low). ${ }^{40} \mathrm{PET}$ permits earlier diagnosis of bone metastases in MM, showing bone resorption sites undetected with conventional diagnostic methods. In addition, it can reveal metastatic spread to sites other than bone. ${ }^{41}$

\section{Treatment}

Treatment decisions depend on several parameters, for example, if bone disease is localized or widespread, if there is evidence of extraskeletal metastases, the kind of cancer and his features (like estrogen-receptors in $\mathrm{BC}$ ), prior treatment history and disease response, the symptoms and the general state of health. ${ }^{28}$ Treatments can often shrink or slow the growth of bone metastases and can help with the symptoms they are causing but, they are not curative.

Bisphosphonates are analogues of pyrophosphate, a natural inhibitor of bone demineralization. ${ }^{28}$ Bisphosphonates bind avidly to exposed bone mineral around resorbing osteoclast and this leads to very high local concentrations of product in the resoption lacunae. Then, bisphosphonates are internalized by the osteoclast causing disruption of the chemical process involved in bone resorption. ${ }^{5,42}$ Bisphosphonates also cause osteoclast apoptosis and some studies suggest that they may also have direct apoptotic effects on tumour cells. ${ }^{5,43}$ In oncology, bisphosphonates are the standard treatment for tumour-induced hypercalcaemia and a new form of therapy for bone metastasis. ${ }^{44}$

With intravenous bisphosphonates and rehydration, $70-90 \%$ of patients will achieve normocalcaemia. The effect on pain in bone metastases is independent of the nature of the underlying tumour and the sclerotic lesions respond similarly to lytic metastases. ${ }^{45}$ The studies are mainly done in BC and MM; lung, kidney and prostatic cancer have few studies.

They are well tolerated. The most common adverse events include flu-like symptoms (fever, arthralgia, myalgia and weakness), anemia, nausea, dyspnea and peripheral edema. These events are mostly limited and mild to moderate. ${ }^{5}$ A rare but very serious side effect is osteonecrosis of the jaw. All bisphosphonates undergo renal clearance so, patients with renal impairment (serum creatinine level $>3.0 \mathrm{mg} / \mathrm{dL}$ ) should not receive the treatment. ${ }^{46}$

There are three generations of bisphosphonates: $1^{\text {st }}$ generation, etidronate, clodronate, tiludronate; $2^{\text {nd }}$ generation, pamidronate, alendronate, ibandronate; $3^{\text {rd }}$ generation, risedronate, zoledronic acid. The approved ones are: oral clodronate at a daily dose of $1600 \mathrm{mg}$ and oral ibandronate $50 \mathrm{mg}$; intravenous (IV) pamidronate $90 \mathrm{mg}$ (infusion of $2 \mathrm{~h}$ ), ibandronate $6 \mathrm{mg}$ (infusion of $1 \mathrm{~h}$ ), zoledronic acid $4 \mathrm{mg}$ (infusion of $15 \mathrm{~min}$ ). Zoledronic acid is the newer bisphosphonate approved for MM, lung, prostate and $\mathrm{BC}$ with bone metastasis. It is 100-times more effective than pamidronate. ${ }^{47}$ Patients doing these treatments should take a supplement containing calcium and vitamin D.

Bisphosphonates, besides the beneficial effects on pain and reduction of fractures, also display antimyeloma and antitumor activity with prolonged overall survival reported for various malignancies. ${ }^{48-51}$ Bisphosphonates exert their antitumor effects by interrupting the vicious cycle of increased osteolysis coupled with increased tumor growth. With this action, bisphosphonates may preserve bone health and delay bone lesion progression. Direct effects of bisphosphonates on cancer cells may contribute to the antitumor effect. For example, zoledronic acid inhibits growth, migration, and matrix-associated invasion of BC cells. In vitro, BC cells treated with ibandronate showed attenuated proliferation. ${ }^{52}$ Bisphosphonates may induce apoptosis in neoplastic cells via modulation of the activity of small GTPases (GTPases downregulate the expression of proapoptotic genes in malignant cells, and bisphosphonates inhibit the activity of small GTPases). ${ }^{53}$ At last, bisphosphonates may stimulate innate antitumor immune mechanisms. In patients with prostate cancer, zoledronate therapy elicited a long-term shift of peripheral $\mathrm{T}$ cells towards an activated effector memory-like state associated with improved immune surveillance against malignant cells. ${ }^{54}$

A meta-analysis that included 17 trials with 1520 patients analyzed in bisphosphonates groups, and 1490 analyzed in control groups, found no significant effect of bisphosphonates on overall survival and progression-free survival. ${ }^{55}$ On the other hand, zoledronic acid demonstrated disease-free survival benefits and a $15 \%$ improvement in overall survival in a metaanalysis including 9518 BC patients. ${ }^{56}$ We conclude that information is very contradictory but beneficial seems to be obvious.

Denosumab is a human monoclonal antibody that inhibits the RANKL, preventing the development of osteoclasts. It can help prevent or delay problems like fractures in patients with bone metastases at least as well as zoledronate, and is safe to give to patients with impaired renal function. It also can be helpful when zoledronate is no longer working. ${ }^{57}$ It is injected subcutaneously. Dosing ranges from $60 \mathrm{mg}$ every 6 months in order to preserve bone density in postmenopausal women to $120 \mathrm{mg}$ every 4 weeks for malignant disease metastatic to the bone. Denosumab does not accumulate in the bone as bisphosphonates, and its effect is reversible after treatment discontinuation. ${ }^{58}$ Side effects are similar to bisphosphonates, including nausea, diarrhea, weakness and can cause osteonecrosis of the jaw too. ${ }^{57}$

Osteonecrosis of the jaw is more common when IV bisphosphonates or denosumab are administered on a monthly basis for 
control of metastases and is much less frequent with less intensive use of bisphosphonates or denosumab for preservation of bone mass. Osteonecrosis of the jaw management is mostly conservative, and healing occurred in more than one-third of patients. Most of the patients with confirmed osteonecrosis of the jaw had a history of tooth extraction (62\%), poor oral hygiene and/or use of a dental appliance..$^{59}$

Radiotherapy is the treatment of choice for localized bone pain, but in presence of poorly localized bone pain or recurrence of pain in previously irradiated skeletal sites, the bisphosphonates are an alternative treatment approach. ${ }^{28}$

The main benefits of denosumab are the possibility to be used in renal failure (denosumab clearance is independent of renal function, in contrast to bisphosphonates clearance, since denosumab is cleared by reticuloendothelial system); ${ }^{60}$ the reversibility of its effect after treatment discontinuation; acute-phase reactions occur rarely after denosumab (but are frequent after zoledronic acid); in prostate and BC patients, suppression of bone turnover markers is greater than by bisphosphonates. ${ }^{61,62}$ The weaknesses of denosum$\mathrm{ab}$ are the increased infection rate in patients with osteoporosis or early $\mathrm{BC} ; 63$ the post-market period of denosumab is still comparably short and yet unknown side effects may emerge; in lung and MM cancer patients it is equipotent to bisphosphonates in preventing skeletal-related events, ${ }^{64}$ it was showed a worse survival in patients with MM treated with denosumab compared to zoledronate; ${ }^{64}$ and the economic burden.

External radiotherapy provides excellent palliation for localized metastatic bone pain however, ${ }^{65}$ the mechanism of pain relief after radiation therapy is poorly understood. ${ }^{28}$ Pain relief usually occurs rapidly, with more than $50 \%$ of responders showing benefit within 1-2weeks. If improvement in pain has not occurred by 6 weeks or more after treatment, it is unlikely to be achieved. ${ }^{33}$ Indications for radiotherapy for bone metastases include pain, risk for pathologic fracture and neurological complications arising from spinal cord compression. ${ }^{5}$

Radiation therapy can be delivered using three forms of treatment: local-field radiation therapy, wide-field radiation therapy and radionuclide therapy. ${ }^{28}$ The local-field radiation therapy is considered the conventional treatment of bone metastases. It treats the involved bone and yields a pain relief rate of $80-90 \%{ }^{66}$ Several randomized trials have indicated that a single fraction of $8 \mathrm{~Gy}$ is adequate for pain relief. ${ }^{65}$ Wide field (half-body, hemibody) radiation therapy can be used as primary palliative therapy for widespread symptomatic bone metastases or as an adjuvant to localfield radiation to reduce the later expression of occult metastases and to reduce the frequency of re-treatment. ${ }^{67,68}$ It is possible to distinguish: upper wide-field treatments (from skull or C1 to L2-3) - optimal single-dose is 6Gy; mid-body wide-field treatments (from L1 to upper third of the femurs) - optimal single-dose is 8Gy; lower wide-field treatments (from L3-4 to above the knees) optimal single-dose is $8 \mathrm{~Gy} .{ }^{66}$ Wide-field radiation provides pain relief for $64-100 \%$ of patients and approximately $50-66 \%$ of patients maintain pain relief for the remainder of their lives. The radiation fields must be shaped to reduce exposure of sensitive structures such as lung, gut, kidney and liver.

Radionuclide therapy is the systemic use of radioisotopes for bone pain. ${ }^{28}$ Radiopharmaceuticals like strontium-89, rhenium186 or samarium-153, have been shown to be effective in palliation of metastatic bone pain. They are preferentially taken up at sites for bone formation, so they probably are most effective for osteoblastic metastases. ${ }^{69}$ The principal side effects are myelosuppression and pain flare.

Recently we have the radium-223, calcium mimetic and alpha emitter that selectively binds to areas of increased bone turnover in bone metastases. It bounds into newly formed bone stroma and the radiation induces mainly double-stranded DNA breaks that result in a potent and highly localized cytotoxic effect. Toxic effects on adjacent tissues and particularly the bone marrow are minimal due to the short path of the alpha particles. Radium-223 significantly prolonged overall survival in patients who had castration-resistant prostate cancer and bone metastases, with a $30 \%$ reduction in the risk of death. ${ }^{70}$

Ablation is the procedure where a needle or probe is introduced into a tumor and using heat, cold or a chemical, the tumor is destroyed. It may be used if only 1 or 2 bone tumors are causing symptoms. The most common types of ablation are radiofrequency ablation (RFA), where an electric current delivered through the needle heats the tumor to destroy it; and cryoablation, where a very cold probe is put into the tumor to freeze the cancer cells. ${ }^{71}$ Although effective at reducing a patient's pain, RFA has a critical limitation, which is the nonvisualization of the ablation margin with CT monitoring. In contrast to RFA, the ablation zone of cryoablation is readily identified with CT imaging as a low-attenuation ice ball beyond which tissues are safe from thermal injury. ${ }^{72}$ Another advantage of cryoablation relative to RFA is that patients treated with cryoablation do not experience increased pain during the procedure or in the immediate posttreatment period. ${ }^{73}$ Although the complication rate using RFA and cryoablation for treatment of painful metastases is low, it was reported neurologic injuries, neuropathic pain and infection in the treatment area. ${ }^{74}$ For systemic antitumor treatment selection, the pathological type of the tumor is most important. In lymphoma and germ cell tumors involving bone chemotherapy can be curative, while in renal cell carcinoma or melanoma it has little effect. ${ }^{3}$

Recently, there are cabozantinib (XL184), an oral tyrosine kinase inhibitor whose targets include VEGFR2, MET, KIT, and mutationally activated RET. It was associated with high rates of bone scan improvement, but the side effects required dose reduction or treatment discontinuation. In addition, statistically significant improvement in progression-free survival was seen with cabozantinib compared with placebo, in metastatic prostate cancer. $^{75}$

Surgery is only indicated for fractures of long bones and hip joints, in spinal cord involvement, or peripheral nerve compression. ${ }^{5}$

Stereotactic radiosurgery has emerged as a new treatment option for the multidisciplinary management of metastases located within or adjacent to vertebral bodies and the spinal cord. The goals of stereotactic radiosurgery are to improve local control over conventional fractionated radiation therapy and to be effective for the treatment of previously irradiated lesions with an acceptable safety profile. Stereotactic radiosurgery offers several theoretical advantages as a treatment modality for spinal tumors: early treatment of these lesions before a patient becomes symptomatic and the stability of the spine, it avoids the need to irradiate large segments of the spinal cord, the early treatment of spinal lesions may obviate the need for extensive spinal surgery for decompression and fixation in these already debilitated patients and may also avoid the need to irradiate large segments of the spinal column, which is known to have a deleterious effect on bone marrow reserve in these patients. The avoidance of open surgery and the preservation of bone-marrow function facilitate continuous chemotherapy in this patient population. Other advantage is that treatment can be completed in a single day rather than over the course of several weeks. The limitations of stereotactic radiosurgery for spinal metastasis are: the quality of literature is poor; no randomized controlled study has been conducted; stereotactic radiosurgery is more expensive than conventional $\mathrm{RT} .{ }^{76,77}$ 
Assessing the response of bone metastases to therapy is difficult; the events in the healing process are slow to evolve and quite subtle, with sclerosis of lytic lesions only beginning to appear 3-6 months after the start of therapy and taking more than a year to mature. It is generally accepted that sclerosis of lytic metastases with no radiological evidence of new lesions constitutes tumor regression (a partial response). Confounding factors include the appearance of sclerosis in an area that was previously normal. After successful therapy for metastatic disease, the healing processes of new bone formation cause an initial increase in tracer uptake and scans carried out during this phase are likely to show increased production of new bone and isotope uptake gradually falls. ${ }^{33}$

\section{Conclusions}

The presence of bone metastases is a sign of disseminated disease and foretells a short-term prognosis in cancer patients. The bone metastases have an important impact on patient's quality of life so, new strategies are necessary to prevent skeletal disease and palliate established skeletal events. A multidisciplinary approach should include medical oncologist, radiotherapist, pain control team, intervention radiologist, endocrinologist, orthopedic surgeon and psychologist.

More studies are necessary to elucidate the interaction between tumor cell and bone microenvironment to reach new therapeutic interventions.

\section{References}

1. Chambers A, Naumov G, Varghese H, et al. Critical steps in hematogenous metastasis: an overview. Surg Oncol Clin N Am 2001;10:243-55.

2. Luzzi KJ, MacDonald IC, Schmidt EE, et al. Multistep nature of metastatic inefficiency: dormancy of solitary cells after successful extravasation and limited survival of early micrometastases. Am J Pathol 1998;153:865-73.

3. Coleman R. Metastatic bone disease: clinical features, pathophysiology and treatment strategies. Cancer Treat Rev 2001;27:165-76.

4. Cecchini M, Wetterwald A, Pluijm G, Thalmann G. Molecular and biological mechanisms of bone metastasis. EAU Update Series 2005;3:214-26.

5. Selvaggi G, Scagliotti G. Management of bone metastases in cancer: a review. Clin Rev Oncol Hematol 2005;56:365-78.

6. Taube T, Elomaa I, Blomqvist C. Histomorphometric evidence for osteoclast-mediated bone resorption in metastatic breast cancer. Bone 1994;15:161.

7. Southby J, Kissin M, Danks J, et al. Immunohistochemical localization of parathyroid hormone-related protein in human breast cancer. Cancer Res 1990;50:7710-6.

8. Kohno N, Kitazawa S, Fukase M, et al. The expression of parathyroid hormone-related protein in human breast cancer with skeletal metastases. Surg Today 1994;24:215-20.

9. Dougall W, Glaccum M, Charrier K, et al. Rank is essential for osteoclast and lymph node development. Genes Dev 1999;13:2412-24.

10. Keller E, Zhang J, Cooper C, et al. Prostate carcinoma skeletal metastases: cross-talk between tumor and bone. Cancer Metastasis Rev 2001;20:333-49.

11. Iwamura M, Hellman J, Cockett A, et al. Alteration of the hor- monal bioactivity of parathyroid hormone-related protein (PTHrP) as a result of limited proteolysis by prostate-specific antigen. Urology 1996;48:317-25.

12. Yang X, Karsenty G. Transcription factors in bone: developmental and pathological aspects. Trends Mol Med 2002;8:3405.

13. Coleman R, Seaman J. The role of zoledronic acid in cancer: clinical studies in the treatment and prevention of bone metastasis. Semin Oncol 2001;28:11-6.

14. Berrettoni BA, Carter JR. Mechanisms of cancer metastasis to bone. J Bone Joint Surg Am 1986;68:308-12.

15. Franks LM. The spread of prostatic carcinoma to the bones. $J$ Pathol Bacteriol 1953;66:91-3.

16. Springer TA. Traffic signals for lymphocyte recirculation and leukocyte emigration: the multistep paradigm. Cell 1994;76:301-14.

17. Mazo IB, von Andrian UH. Adhesion and homing of bloodborne cells in bone marrow microvessels. J Leukoc Biol 1999;66:25-32.

18. Sipkins DA, Wei X, Wu JW, et al. In vivo imaging of specialized bone marrow endothelial microdomains for tumour engraftment. Nature 2005;435:969-73.

19. Balkwill F. Cancer and the chemokine network. Nat Rev Cancer 2004;4:540-50.

20. Guo W, Giancotti FG. Integrin signalling during tumour progression. Nat Rev Mol Cell Biol 2004;5:816-26.

21. Russel H, Hicks J, Okcu M, Nuchtern J. CXCR4 expression in neuroblastoma primary tumors is associated with clinical presentation of bone and bone marrow metastases. J Pediatr Surg 2004;39:1506-11.

22. Jones D, Nakashima T, Sanchez O, et al. Regulation of cancer cell migration and bone metastasis by RANKL. Nature 2006;440:692-6.

23. Papotti M, Kalebic T, Volante M, et al. Bone sialoprotein is predictive of bone metastases in resectable non-small-cell lung cancer: a retrospective case-control study. J Clin Oncol 2006;24:4818-24.

24. Paget $\mathrm{S}$. The distribution of secondary growths in cancer of the breast. Lancet 1889;1:571-3.

25. Coleman R, Rubens R. The clinical course of bone metastases from breast cancer. Br J Cancer 1987;55:61-6.

26. Coleman R, Smith P, Rubens R. Clinical course and prognostic factors following recurrence from breast cancer. $\mathrm{Br} \mathrm{J}$ Cancer 1998; 17:336-40.

27. Robson M, Dawson N. How is androgen dependent metastatic prostate cancer best treated? Haematol Oncol Clinics North Am 1996;10:727-47.

28. Maisano R, Pergolizzi S, Cascinu S. Novel therapeutic approaches to cancer patients with bone metastasis. Crit Rev Oncol Hematol 2001;40:239-50.

29. Higinbotham N, Marcove R. The management of pathological fractures. J Trauma 1965;5:792-9.

30. Nielson O, Munro A, Tannock I. Bone metastases: pathophysiology and management policy. J Clin Oncol 1991;9:509-24.

31. Hadji P, Aapro M, Body J, et al. Management of aromatase inhibitor-associated bone loss in postmenopausal women with breast cancer: practical guidance for prevention and treatment. Ann Oncol 2011;22:2546-55.

32. Clohisy D, Mantyh P. Bone cancer pain. Cancer 2003;97:866.

33. Coleman R, Body J, Aapro M, et al. Bone health in cancer patients: ESMO clinical practice guidelines. Ann Oncol 2014;25:124-37.

34. Algra P, Bloem J, Tissing H, et al. Detection of vertebral metastases: comparison between MRI and bone scintigraphy. 
Radiographics 1991;11:219-32.

35. Vinholes J, Coleman R, Eastell R. Effects of bone metastases on bone metabolism: implications for diagnosis, imaging and assessment of response to cancer treatment. Cancer Treat Rev 1996;22:289-31.

36. Rosenthal D. Radiologic diagnosis of bone metastases. Cancer 1997;80:1595-607.

37. Evans A, Robertson J. Magnetic resonance imaging versus radionuclide scintigraphy for screening in bone metastases. Clin Radiol 2000;55:653.

38. Marom E, McAdams H, Erasmus J, et al. Staging non-small cell lung cancer with whole-body PET. Radiology 1999;212:803-9.

39. Yang S, Liang J, Lin F, et al. Comparing whole-body 18 FDG PET and technetium-99m methylene diphosphonate bone scan to detect bone metastases in patients with breast cancer. J Cancer Res Clin Oncol 2002;128:325-8.

40. Schirrmeister H, Guhlmann A, Elsner K, et al. Sensitivity in detecting osseous lesions depends on anatomic localization: planar bone scintigraphy versus $18 \mathrm{~F}$ PET. J Nucl Med 1999;40:1623-9.

41. Shreve P, Grossman H, Gross M, Wahl R. Metastatic prostate cancer: initial findings of PET with 2-deoxy-2 (F-18) fluoro-dglucose. Radiology 1996;199:751-5.

42. Rogers M, Xiong X, Ji X, et al. Inhibition of growth of dictyostelium discoideum amoebae by bisphosphonates is dependent on cellular uptake. Pharmacol Res 1997;14:625-30.

43. Rogers M, Watts D, Russel R. Overview of bisphosphonates. Cancer 1997;80:1652-60.

44. Body J, Coleman R, Piccart M. Use of bisphosphonates in cancer patients. Cancer Treat Rev 1996;22:265-87.

45. Vinholes J, Purohit O, Abbey M, et al. Relationships between biochemical and symptomatic response in double-blind trial of pamidronate for metastatic bone disease. Ann Oncol 1997;8:1243-50.

46. Green JR, Rogers MJ. Pharmacologic profile of zoledronic acid: a highly potent inhibitor of bone resorption. Drug Dev Res 2002;55:210-24

47. Wellington K, Goa KL. Zoledronic acid. A review of its use in the management of bone metastases and hypercalcemia of malignancy. Drugs 2003;63:417-37.

48. Guenther A, Gordon S, Tiemann M, et al. The bisphosphonate zoledronic acid has antimyeloma activity in vivo by inhibition of protein prenylation. Int J Cancer 2010;126:239-46.

49. Rennert G, Pinchev M, Rennert H, Gruber S. Use of bisphosphonates and reduced risk of colorectal cancer. J Clin Oncol 2011;29:1146-50.

50. Rennert G, Pinchev M, Rennert HS. Use of bisphosphonates and risk of postmenopausal breast cancer. J Clin Oncol 2010;28:3577-81.

51. Chlebowski R, Chen Z, Cauley J, et al. Oral bisphosphonate use and breast cancer incidence in postmenopausal women. $\mathrm{J}$ Clin Oncol 2010;28:3582-90.

52. Dedes P, Gialeli C, Tsonis A, et al. Expression of matrix macromolecules and functional properties of breast cancer cells are modulated by the bisphosphonate zoledronic acid. Biochim Biophys Acta 2012;1820:1926-39.

53. Iguchi K. Effect of bisphosphonates on anticancer activity in prostate cancer cells. Yakugaku Zasshi 2012;132:1025-30.

54. Aapro M, Saad F. Bone-modifying agents in the treatment of bone metastases in patients with advanced genitourinary malignancies: a focus on zoledronic acid. Ther Adv Urol 2012;4:85-101.

55. Mhaskar R, Redzepovic J, Wheatley K, et al. Bisphosphonates in multiple myeloma. Cochrane Database Syst Rev 2010;17:CD003188.

56. Huang W, Huang C, Lui J, et al. Zoledronic acid as an adjuvant therapy in patients with breast cancer: a systematic review and meta-analysis. PLoS One 2012;7:e40783.

57. Steger G, Bartsch R. Denosumab for the treatment of bone metastases in breast cancer: evidence and opinion. Ther Adv Med Oncol 2011;3:233-43.

58. Yee A, Raje N. Denosumab, a RANK ligand inhibitor, for the management of bone loss in cancer patients. Clin Interv Aging 2012;7:331-8.

59. Migliorati C, Epstein J, Abt E, Berenson J. Osteonecrosis of the jaw and bisphosphonates in cancer: a narrative review. Nat Rev Endocrinol 2011;7:34-42.

60. Baron R, Ferrari S, Russell R. Denosumab and bisphosphonates: different mechanisms of action and effects. Bone 2011;48:677-92.

61. Stopeck A, Lipton A, Body J, et al. Denosumab compared with zoledronic acid for the treatment of bone metastases in patients with advanced breast cancer: a randomized, doubleblind study. J Clin Oncol 2010;28:5132-9.

62. Fizazi K, Carducci M, Smith M, et al. Denosumab versus zoledronic acid for treatment of bone metastases in men with castration resistant prostate cancer: a randomised, double-blind study. Lancet 2011;377:813-22.

63. Anastasilakis A, Toulis K, Goulis D, et al. Efficacy and safety of denosumab in postmenopausal women with osteopenia or osteoporosis: a systematic review and a meta-analysis. Horm Metab Res 2009;41:721-9.

64. Henry D, Costa L, Goldwasser F, et al. Randomized, doubleblind study of denosumab versus zoledronic acid in the treatment of bone metastases in patients with advanced cancer (excluding breast and prostate cancer) or multiple myeloma. J Clin Oncol 2011;29:1125-32.

65. Janjan N. Radiation of bone metastases: conventional techniques and the role of systemic radiopharmaceuticals. Cancer 1997;80:1628-45.

66. Tong C, Gillick L, Hendrickson F. The palliation of symptomatic osseous metastases: final results of the study by the Radiation Therapy Oncology Group. Cancer 1982;50:893-9.

67. Salazar O, Rubin P, Hendrickson F, et al. Single-dose halfbody irradiation for palliation of multiple bone metastases from solid tumors: final Radiation Therapy Oncology Group report. Cancer 1986;58:29-36.

68. Poulter C, Cosmatos D, Rubin P, et al. A report of RTOG 8206: a phase III study of whether the addition of single dose hemibody irradiation to standard fractionated local field irradiation is more effective than local field irradiation alone in the treatment of symptomatic osseous metastases. Int J Radiat Oncol Biol Phys 1992;23:207-14.

69. Robinson R, Preston D, Baxter K, et al. Clinical experience with strontium 89 in prostatic and breast cancer patients. Semin Oncol 1993;20:44-8.

70. Parker C, Nilsson S, Heinrich D, et al. Alpha emitter Radium223 and survival in metastatic prostate cancer. N Engl J Med 2013;18:213-23.

71. American Cancer Society. Bone metastasis. Available from: www.cancer.gov

72. Saliken J, McKinnon J, Gray R. CT for monitoring cryotherapy. AJR Am J Roentgenol 1996;166:853-5.

73. Thacker P, Callstrom M, Curry T, et al. Palliation of painful metastatic disease involving bone with image-guided treatment: comparison of patients immediate response to radiofrequency ablation and cryoablation. AJR Am J Roentgenol 
2011;197:510-5.

74. Callstrom M, Dupuy D, Solomon S, et al. Percutaneous imageguided cryoablation of painful metastases involving bone: multicenter trial. Cancer 2013;119:1033-41.

75. Lee R, Saylor P, Michaelson M, et al. A dose-ranging study of cabozantinib in men with castration-resistant prostate cancer and bone metastases. Clin Cancer Res 2013;19:3088-94.

76. Jhaveri $\mathrm{P}$, Teh B, Bloch $\mathrm{C}$, et al. Stereotactic body radiotherapy in the management of painful bone metastases. Oncology 2008;22:782-8.

77. Sohn S, Chung C. The role of stereotactic radiosurgery in metastasis to the spine. J Korean Neurosurg Soc 2012;51:1-7. 